\title{
Analysis of Particulate Contamination During Launch of MMS Mission
}

\author{
Lubos Brieda, Alexander Barrie \\ Millenium Engineering and Integration, Arlington, VA \\ David Hughes, Therese Errigo \\ NASA Goddard Space Flight Center, Greenbelt,MD
}

\begin{abstract}
NASA's Magnetospheric MultiScale (MMS) is an unmanned constellation of four identical spacecraft designed to investigate magnetic reconnection by obtaining detailed measurements of plasma properties in Earth's magnetopause and magnetotail. Each of the four identical satellites carries a suite of instruments which characterize the ambient ion and electron energy spectrum and composition. Some of these instruments utilize microchannel plates and are sensitive to particulate contamination. In this paper, we analyze the transport of particulates during pre-launch, launch and ascent events, and use the analysis to obtain quantitative predictions of contamination impact on the instruments. Viewfactor calculation is performed by considering the gravitational and aerodynamic forces acting on the particles.
\end{abstract}

Keywords: particulate contamination, Magnetospheric Multiscale Mission (MMS), mass transport

\section{INTRODUCTION}

This paper presents results from a particulate contamination analysis performed in support of the NASA Magnetospheric Multiscale Mission (MMS). MMS is a constellation of four identical satellites designed to investigate magnetic reconnection in Earth's magnetopause and is scheduled to launch in 2014. Magnetic reconnection is a fundamental plasma process in which potential energy stored in the magnetic field is converted into kinetic energy, resulting in high energy electron beams, solar flares and auroral discharges. In order to investigate this process, each satellite carries an extensive suit of instruments designed to measure the ambient electromagnetic environment and to characterize the charged particle populations. Spacecraft design, testing and integration are being performed at the Goddard Space Flight Center in Greenbelt, Maryland. The science investigation is lead by a team of researchers at the Southwest Research Institute (SwRI) in San Antonio, Texas ${ }^{1}$.

The particle instruments utilize an electrostatic analyzer (ESA) to filter populations by energy and microchannel plates (MCPs) to increase signal strength. MCPs are signal multipliers that use plates containing a large number of channels coated with a material possessing a high secondary electron yield. These instruments are highly sensitive to particulate contamination ${ }^{2}$, due to the precise electrostatic state required by the ESA and the surface uniformity required by the MCP. As such, it became imperative to analyze the risk to the instruments from contaminant fallout during events preceding orbit insertion. The spacecraft experiences a wide range of environments after encapsulation, including the flow of air conditioning air used in thermal control, acceleration, vibrational and acoustic loads during launch, and depressurization venting during ascent. The applied forces associated with these processes transport particles to locations outside the direct line of sight of the contamination source. The typical line of sight redistribution analysis assuming grey-body analysis, was therefore not sufficient. Integration of equations of motion was required. In this paper, we completed such an analysis. We used an in-house mass transport code to calculate particle motion in several representative environments. Transport results were combined with particle detachment model to predict cleanliness levels on the instruments of interest.

\section{MISSION OVERVIEW}

Each of the four satellites consists of an octagonal bus built around a central cylindrical tube containing the propellant tanks. The bus is approximately 11 feet wide and 4 feet high and the eight sides house the body-mounted solar panels. The primary instrument suite consists of sensors mounted on the underside of the top deck. Magnetometer booms, designed to investigate the magnetic field, are mounted on the underside of the bottom deck. The bottom deck also houses one of the two particle spectrometers known as FEEPS. Schematic of the satellite design is shown in Figure 1. 

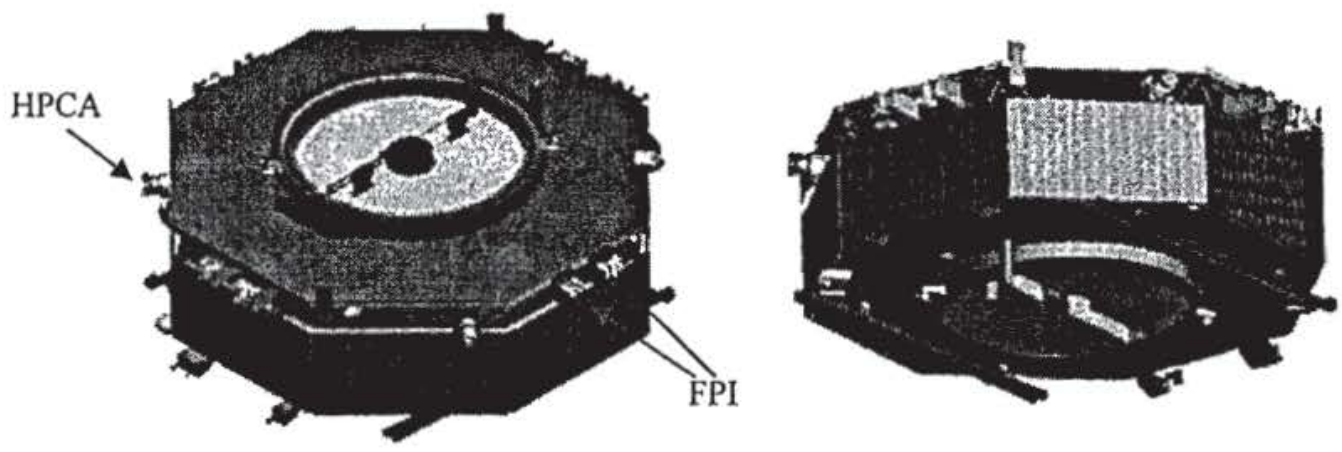

Figure 1. Single MMS constellation spacecraft. Thermal blankets not shown. The figure shows the location of the HPCA and one of the four FPI instruments. Figure from Ref. 1.

The four satellites will be launched into orbit in a stacked configuration using a single Atlas 400 series launch vehicle. The spacecraft will be inserted into orbit sequentially. As can be seen in Figure 2, only a narrow gap exists between the launch vehicle payload envelope and the instrument suite which extends past the solar panels. This narrow gap, coupled with surface irregularities, is expected to generate turbulent air flow which may transport particles into regions with no direct line of sight of the originating source. Alternating spacecraft are clocked $180^{\circ}$ from each other.

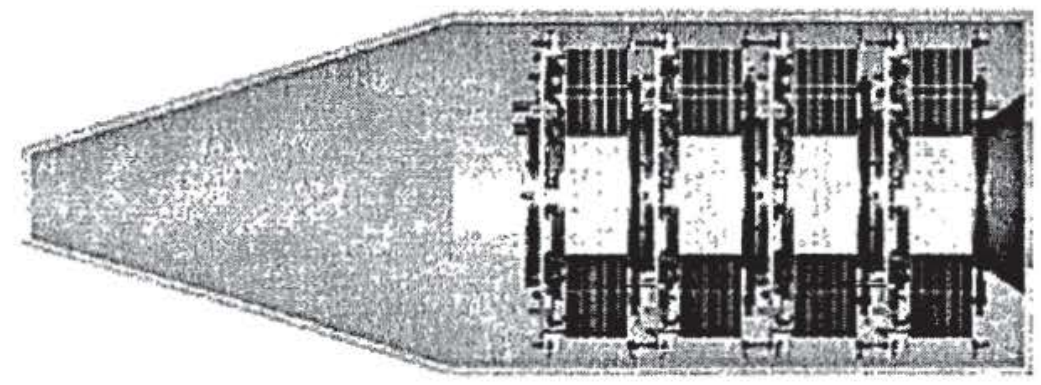

Figure 2. Launch configuration of the four MMS spacecraft.

\subsection{Instrument Suite}

The science payload consists of an instrument suite comprised of nine types of instruments. These instruments can be grouped into three main categories based on their primary objective: hot plasma, energetic particles, and fields. The first and second category includes devices designed to measure energy spectrum and composition of the incoming charged particles, but differ in the energy regimes considered. Both of these groups are contamination sensitive. The fields group includes probes that measure evolution of the ambient electromagnetic fields. The probes used in the fields suite are largely insensitive to contamination effects, aside from the Electron Drift Instrument (EDI).

The first category measures composition of particles in the $10 \mathrm{eV}$ to $30 \mathrm{keV}$ range, and include the Fast Plasma Investigation (FPI) and Hot Plasma Composition Analyzer (HPCA). FPI collects full-sky, 3D images of the ambient plasma using two sensors, the Dual Electron Spectrometer (DES) and the Dual Ion Spectrometer (DIS). There are four FPI pairs placed around the spacecraft at $90^{\circ}$ intervals. HPCA utilizes an RF circuit and a time-of-flight analysis to characterize hydrogen, helium and oxygen ions. Both instruments use hemispherical electrostatic analyzers ${ }^{3}$ for energy spectrum analysis and MCPs.

The sensors in the second category, the energetic particles group, investigate particle fluxes in the $45 \mathrm{keV}$ to $500 \mathrm{keV}$ range. These sensors include Fly's Eye Particle Sensor (FEEPS) and Energetic Ion Spectrometer (EIS). The two FEEPS instruments on each spacecraft measure 3D energetic ion and electron flux distributions at time resolution of 10 seconds. EIS uses a time-of-flight sensor to obtain ion composition and angular distribution measurements with temporal resolution of $30 \mathrm{~s}$. Both instruments use MCPs.

The fields group includes several double probes and magnetometers, as well as the Electron Drift Instrument (EDI). This group measures the electric and magnetic fields around the spacecraft. The twin EDI instruments located on each 
spacecraft measure the electron gyrofrequency by detecting a coded electron beam emitted by the other instrument's injection unit (GDU). Microchannel plates are used on EDI. Electric potential on the spacecraft is neutralized by two Active Spacecraft Potential Control Devices (ASPOC).

\subsection{Contamination Concerns}

Figure 3 shows a schematic drawing of MMS particle instruments. Although each instrument is optimized for its specific objective, the instruments utilize similar conceptual operation. Particles enter the electrostatic analyzer through an aperture open to the ambient environment, and the particles with "correct" energy reach the MCPs. Incoming signal is multiplied and current is collected by a segmented anode.

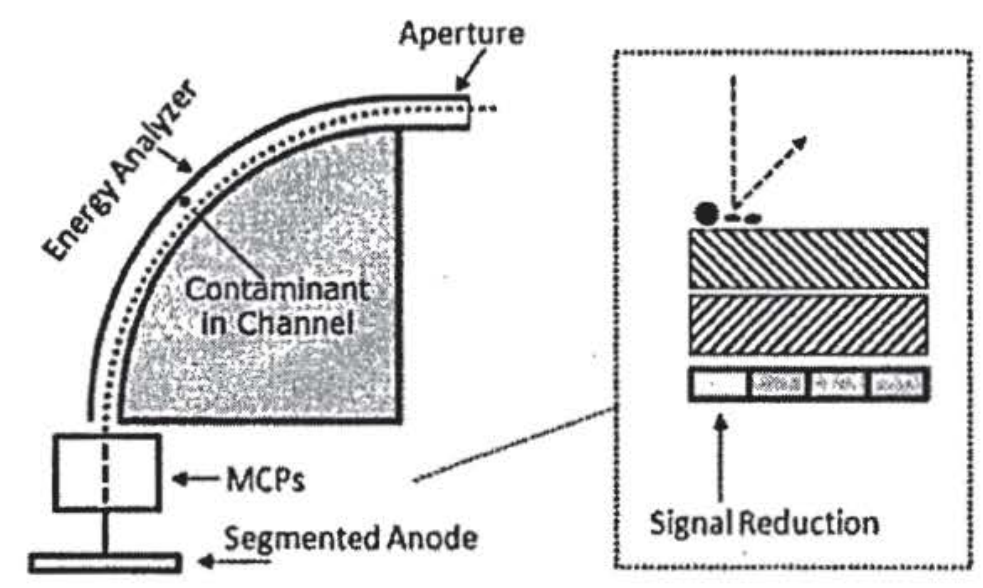

Figure 3. Schematic drawing of MMS particle instruments. Red dashed line indicates the trajectory of an incoming ion or electron. Particulate contamination shown in the ESA channel and on the MCP.

MCPs are voltage multipliers which operate by passing the incoming electrons through a surface containing a large number of tiny channels angled to the incoming trajectory vector. The channels are made of material with high secondary electron yield. Incoming particles are guaranteed to strike the channel wall due to the channel inclination and thus generate additional electrons through secondary electron emission (SEE). A voltage drop is applied across the plate to drive the electrons towards the anode. The SEE electrons again collide with the channel, further multiplying the incident signal resulting in a cascade effect. The MMS instruments utilize a chevron configuration, in which two MCPs with channels in opposite direction are stacked on top of each other, to further increase the signal. The channels give the MCPs spatial resolution which can then be measured by an anode.

MCPs are highly sensitive to contamination by both molecular and particulate sources. Molecular contaminants include volatile atmospheric gases, with water vapor posing the greatest risk. Water vapor outgassing from the plates can undergo ionization, with the ions leading to spurious breakdowns and random noise in the collected current. Non-volatile compounds, such as oils and other hydrocarbons, condense on the surface and polymerize into layers with very low secondary electron yields. These layers reduce the effectiveness of the device, since the electron gain depends directly on the emission of secondary electrons. Particulate contaminants on the MCP can obscure some of the channels resulting in a lowered signal in certain parameter regions of the instrument. This effect is directly proportional to the percent area coverage (PAC) of the MCP. A single fiber, hair, large dust particle, etc. could have a measurable effect on the local performance of the MCP.

The channel of the ESA is also sensitive to contamination. ESA surfaces are typically coated with a very low SEE, UV rejection coating. Contamination in the ESA could lead to spurious counts generated by secondary electron emission from the contaminated area, or UV reflection into the MCP. Dielectric particles in the channel will charge up due to incident charged particles. A typical high voltage standoff of $2500 \mathrm{~V} / \mathrm{mm}$ will allow a 50 micron particle to charge up to 125 volts. This is more than ample charge to affect low energy particles trajectory through the channel. Conductive particles, if large enough, can lead to high voltage discharges and arcing in areas of high voltage differential $(>5 \mathrm{kV})$ over short distances $(\sim 2-3 \mathrm{~mm})$.

In our analysis, we concentrated on the contamination effect on the FPI and HPCA instruments. The orientation of the aperture on the HPCA was of particular concern. This instrument contains a circular aperture looking vertically up 
launch vehicle. Due to close proximity to the payload fairing, this instrument may be highly susceptible to direct particulate fallout from the fairing. HPCA also contains ultra-thin carbon foil which is sensitive to penetration by highvelocity particles, and to modification of apparent thickness by deposition of fine contaminants.

It should be noted that our model was not detailed enough to investigate the particle transport inside the instruments. Instead, the performed analysis only predicted the contamination level at the aperture of the instrument. In order to directly affect the MCP, the particle needs to travel through the aperture and migrate through the analyzer channel. The diffusion velocity of this Brownian motion induced by ion wall flux remains to be computed as follow up work. As such, the results presented here, correspond to the conservative estimates.

\subsection{Cleanliness Levels}

The cleanliness level at beginning of life for all exposed surfaces is 300 per IEST-STD-CC1246D (IEST-1246D). Instruments have a more stringent pre-launch goal of $100 \mathrm{~A} / 3$ for EDI, FPI, EIS, HPCA and $300 \mathrm{~A} / 3$ for FEEPS, with no particles over 100 micron. In our analysis, we assumed that all surfaces were initially cleaned to level 300 .

\section{PARTICULATE TRANSPORT MODEL}

The problem of determining particulate redistribution inside the launch vehicle and its effect on the cleanliness level of the instruments can be divided into three steps. First, the concentration of particles being launched from the contamination sources must be determined. The well known IEST-1246D model specifies the distribution of particle sizes given a certain cleanliness level. This model indicates that concentration increases exponentially as the particle size decreases. On the other hand, small particles are less likely to come off surface due to increased ratio of adhesion to detachment force strength. Particles unable to become detached do not contribute to contamination redistribution. Next, the viewfactors between source and target surfaces must be determined. The typical approach assumes that particles behave like massless rays and the viewfactors are calculated by considering gray body analysis. However, this approach is valid only in the limit of no applied forces, and in the cases considered here, the equations of motion have to be integrated. This step also involves considering particle-surface interaction, since particles will continue to migrate while the impact velocity exceeds attachment (sticking) velocity. Finally, the viewfactors are combined with the source emission model to estimate the cleanliness levels on the target surfaces. Similar approach was previously explored by Crook ${ }^{4}$.

\subsection{Surface Detachment}

In 1987, Klavins and Lee studied the problem of surface adhesion by considering detachment data obtained by applying gravitational loads in a centrifuge $\mathrm{e}^{4}$. These measurements were performed at loads up to $10^{5} \mathrm{~g}$ and showed large variation in the detachment probability. The variation is expected, since the probability that a particle detaches is strongly influenced by the local surface roughness, particle shape and orientation, as well as environmental effects such as humidity or electrostatic charge. Hence, at best simple estimates of detachment probability can be made.

In their work, the authors found the detachment probability to follow $\phi=\left[1+\operatorname{erf}\left(\log \left(a / a_{m}\right) / \sqrt{2} \sigma_{0}\right)\right] / 2$, where $\Phi$ is the detachment probability given applied acceleration $a$ and the standard deviation $\sigma$ is 1.45 . The parameter $a_{m}$ corresponds to the mean acceleration, and is given by $(85.07 / \mathrm{d})^{4.08}$ for particles smaller than 42 micron and $(52.37 / \mathrm{d})^{13.6}$ otherwise". The detachment probability is plotted in Figure 4. This figure indicates that almost all particles larger than 100 micron will detach given applied acceleration of $1 \mathrm{~g}$ or greater. We used this model to calculate the percentage of detached particles given the IEST-1246D distribution. The applied acceleration corresponds to the combination of launch and aerodynamic loads and was calculated as $a=g_{L}+g_{a}+\frac{\rho v^{2} A}{2 m}$ where the subscripts $L$ and $a$ correspond to the launch and acoustic acceleration loads and $v$ is the mean flow velocity near the surface. $A$ is the particle cross-sectional area (assuming spherical particles) and $m$ is the particle mass.

\footnotetext{
- In the reference work, the fits are given as $(52.37 / \mathrm{d})^{4.08}$ and $(85.07 / \mathrm{d})^{136}$, respectively. However, these relationships do not agree with the results presented in the cited work, which are instead approximated by the expressions given above.
} 


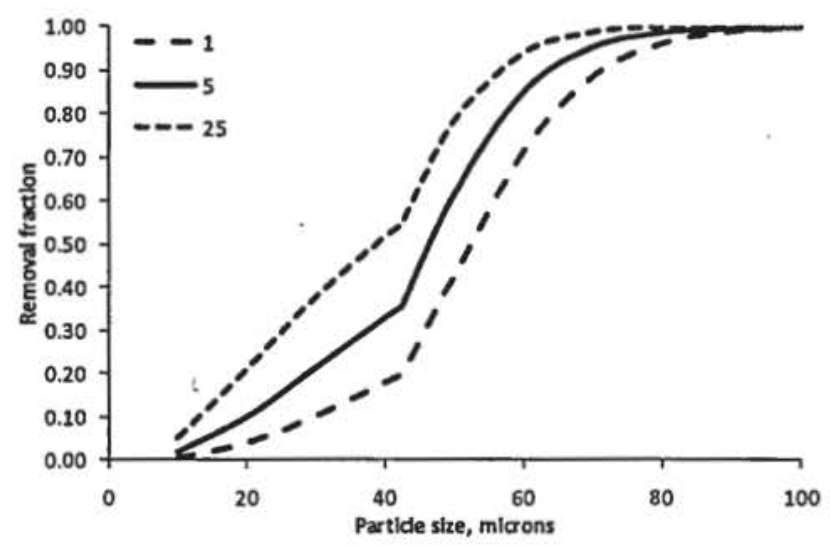

Figure 4. Particle release probability for three selected values of applied force

\subsection{Particle Transport}

Calculation of surface view factors was performed using Mastram, a general purpose tool for spacecraft contamination modeling. Mastram was developed at NASA GSFC by David Hughes and Tony Dazso. The program works by launching a user specified number of test particles from all source elements, and integrating their motion until the particles come to rest or leave the simulation domain. The spacecraft and the fairing are described by a mixed triangular and quadrilateral surface mesh generated by discretizing CAD generated geometry. Siemens NX (Unigraphics) was used for the design and the meshing. The final position of the particles is used to calculate the transport factors (analogous to grey-body view factors) between the source and target element. Mastram includes a graphical user interface which is used to specify material properties, the ambient environment, and the simulation parameters. The code is written in Python, with the particle transport subroutine ported to $\mathrm{C}++$ for enhanced performance.

Motion of individual particles after leaving surface is governed by the equations of motion

$$
\begin{gathered}
\vec{x}^{\prime}=\vec{v} \\
\vec{v}^{\prime}=(1 / m) \sum \vec{F}
\end{gathered}
$$

where the RHS in the second equation includes contribution from all forces acting on the particle. The particle transport governing equations (1) and (2) are integrated using LSODAR, Livermore ODE solver with automatic root finding ${ }^{4}$. The integration is limited by several criterion functions (the roots). The first is a curvature test, $f=\vec{v} \cdot \vec{v}_{0}-|v|\left|v_{0}\right|(1-\delta)$, where $\vec{v}_{0}$ is the velocity at the start of the."time step" and $\delta$ is the tolerance. Although this criterion has no effect on the solution, it is required in order to visualize the curved trajectories of particles. The second test checks for the particle exiting the current bounding box. The efficiency of surface-interaction is improved by subdividing the simulation volume into smaller regions, with each containing a reduced number of surface triangles. The density of the sub-regions is directly proportional to the resolution of the surface mesh. This criterion assures that surface-interaction check is performed against the correct subset of surface triangles. The final criterion tests for collision of particle with surfaces contained in the bounding box. The standard line-triangle intersection algorithm is used.

Forces acting on a particle include gravitational, electrostatic and aerodynamic loads. In our analysis, we assumed that particles do not undergo a significant triboelectric charging, and electrostatic forces could be neglected. The aerodynamic force is given by the drag equation $F_{D}=\frac{1}{2} C_{D} \rho v^{2} A$ where the drag coefficient is the curve-fit of White,

$$
C_{D}=\frac{24}{\operatorname{Re}}+\frac{6}{1+\sqrt{R e}}+0.4 \quad \operatorname{Re}<2 \times 10^{5}
$$

In the drag equation, $v$ is the relative velocity between the particle and the background flow. At every step of the ODE integration, relative velocity is computed by interpolating the background flow velocity onto the particle position. The background flow velocities were assumed to be steady-state, and were obtained from an external CFD solver. Multivariate interpolation based on modified Shepard's method algorithm ${ }^{8}$, as implemented in TOMS Algorithm 661, was used for the calculation. 


\subsection{Surface Interaction}

Upon impacting a surface, some initial kinetic energy of the incoming particle is dissipated in the form of heat. The ratio of the pre- and post-collision velocities is known as the coefficient of restitution. In the simulation, COR is a user defined parameter, and controls the "bounciness" of the particle. A particle with sufficient kinetic energy to overcome the adhesive forces bounces of the surface and Mastram tracks it until another surface impact. This process is repeated until enough kinetic energy is dissipated and the particle comes to rest on a surface. The minimum velocity required to leave the surface is the sticking velocity, and is also a user defined parameter. It was assumed to vary between 0.1 and $0.5 \mathrm{~m} / \mathrm{s}$. The post impact velocity is given by energy balance,

$$
\frac{d}{d t}\left(U+\frac{1}{2} m v^{2}+\frac{1}{2} I \omega^{2}\right)=0
$$

where $U$ is the internal energy corresponding to heat generation in inelastic collision, and $\omega$ is the angular velocity of a rotating particle. The mass of the particle is assumed to remain constant (i.e, the particle does not break up upon impact), and the post bounce velocity is obtained from the coefficient of restitution, $C O R=u_{2} / u_{1}$. Change in rotational energy of spinning particles is calculated from $\Delta I \omega^{2}=m v^{2} M$, where $M$ is the coefficient of momentum transfer ${ }^{8}$.

\subsection{Validation}

We have tested the flow interpolator and path integrator by considering flow between two infinite parallel plates. Particles were injected at $z=0$ at three distances from the bottom plate, and the Mastram trajectories were compared with the theoretical solution. The flow profile is given in any standard aerodynamics text as $u=A\left(a y-y^{2}\right)$, where $A=$ $-(d p / d x) / 2 \mu$. We have taken $a$, the distance between the plates, to be $1 \mathrm{~m}$ and set $A=1(\mathrm{~ms})^{-1}$. The theoretical profile was obtained by integrating the equations of motion, Eqs. 1 and 2, using Matlab's ode 45 ordinary differential equation solver. Figure 5 shows the comparison. Black lines correspond to the particle traces from Mastram and the dashed red lines are the expected theoretical profiles. A very good agreement is seen, with small discrepancies arising due to interpolation error in the rapidly decaying boundary layer.

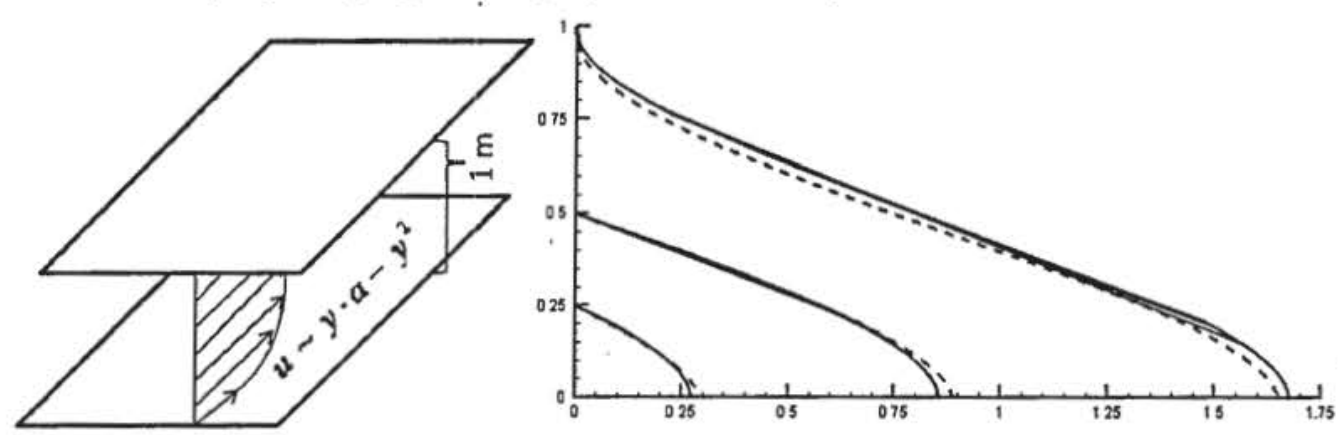

Figure 5. Validation study. The black lines are particle traces from Mastram and the red dashed line indicates the theoretical profile.

\section{SIMULATION INPUTS}

In our investigation, we have considered four characteristic environments, which are encountered by the spacecraft from the time of encapsulation until fairing separation. The cases are outlined in Table 1 . The first case corresponds to the spacecraft loaded in the launch vehicle and sitting on the ground. The air conditioning system is turned on to maintain thermal control and positive pressure gradient with the ambient pressure. Air is injected from an inlet located in the conical section of the fairing in the direction opposite the spacecraft. Ports located above the spacecraft adapter are open to the outside atmosphere.

The second case corresponds to the launch event. The air conditioning system is disconnected and the pressure inside the fairing reaches equilibrium with the ambient sea-level atmosphere. An additional $1 \mathrm{~g}$ launch load acts on the particles and surface detachment is enhanced by the vibrational and acoustic loads. 40 seconds after launch the pressure inside the fairing drops to about $50 \%$ of the sea level value. The vehicle is now accelerating at about $2.5 \mathrm{~g}$. Fluid motion develops inside the fairing due to the depressurization. This configuration corresponds to Case 3. About 4 minutes after liftoff, the vehicle reaches maximum acceleration. The launch vehicle is also leaning over due to the pitch maneuver. We have 
assumed that the vehicle is tilted $30^{\circ}$ from vertical. Final event is the separation of the clamshell. This event differs from the previous four cases in that the primary contamination source is due to particles generated during the violent separation event. This event was not considered in the present work.

Table 1. Summary of the considered cases

\begin{tabular}{|l|l|l|l|l|}
\hline & Air Pressure & Loads & CL Slope & Notes \\
\hline 1. Ground A/C & $>1 \mathrm{~atm}$ & $1 \mathrm{~g}$ & $300 / 0.926$ & $\mathrm{~A} / \mathrm{C}$ \\
\hline 2. Launch & $1 \mathrm{~atm}$, static flow & $3 \mathrm{~g}$ & $\mathrm{TBD} / 0.5$ & Includes $1 \mathrm{~g}$ vibrational load \\
\hline 3. Fairing Venting & $<1 \mathrm{~atm}$ & $3.4 \mathrm{~g}$ & $\mathrm{TBD} / 0.5$ & Venting flow \\
\hline 4. Max Load & $0 \mathrm{~atm}$ & $6 \mathrm{~g}, 30^{\circ}$ tilt & $\mathrm{TBD} / 0.5$ & Prior to burnout \\
\hline
\end{tabular}

\subsection{Launch Vehicle and Satellite ModeI}

The numerical surface mesh used to represent the launch vehicle and the constellation is displayed in Figure 6. Fairing dimensions were obtained from the ULA Mission Planners Guide10. Diagrams in the guide were also used to approximate the location and size of the fairing vents. As can be seen in this figure, the spacecraft geometry was greatly simplified and the magnetometer booms were excluded from the model. We used NX Flow, a CFD add-on to the NX family of CAE tools, to perform the flow analysis. NX Flow includes a volumetric mesher, capable of automatically refining the nodal density in the near-surface boundary layer. The blue elements in Figure 6 show a cut through the volume mesh, with the detail showing the boundary layer mesh in greater detail. The green elements correspond to the surfaces used by Mastram.
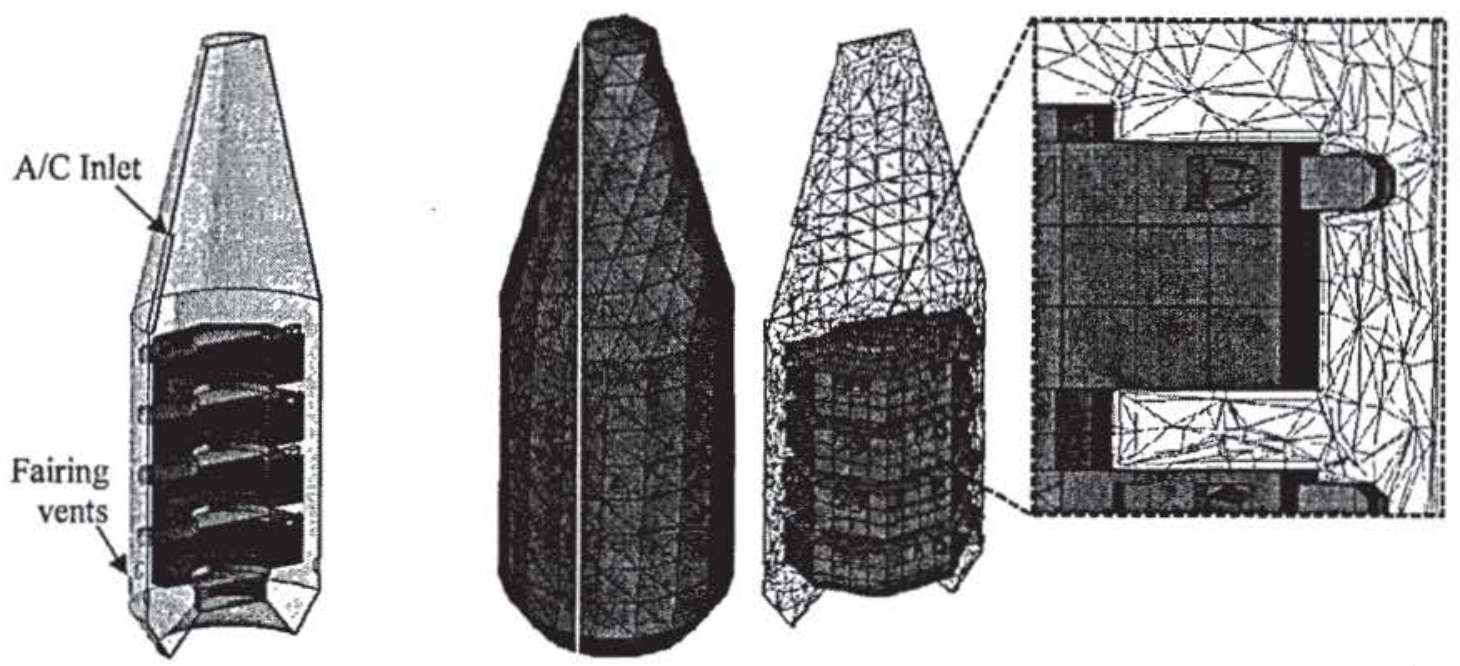

Figure 6. CAD model and the corresponding computational surface mesh (in green). The blue lines show a cut through the computational volume mesh used in the air flow calculation.

The solver includes several turbulence models and can produce both transient and steady-state solutions. In our model, we utilized the mixing length model and surface roughness corresponding to few millimeter sand grains was applied to all surfaces to account for surface irregularities not captured by our design. For the ground air-conditioning case, inlet mass flow rate of $70 \mathrm{~kg} / \mathrm{min}$ was specified on the orifice of the air conditioning hose. The vents were open to the ambient sea-level atmosphere. For the ascent depressurization case, flow velocity $u$ was specified on the fairing vents and was computed from mass conservation and ideal gas law as $\frac{d P}{d t} V=P u A$, where the pressure $P$ and the depressurization rates were obtained from data presented in the MPG for 40 seconds after liftoff. $V$ and $A$, the unoccupied volume insides the fairing and the total vent area, were obtained directly from our model. 
Figure 7 shows the computed flow fields for these two cases. In case 1, the velocity ranges from $50 \mathrm{~m} / \mathrm{s}$ near the air conditioning inlet to about $1 \mathrm{~m} / \mathrm{s}$ at the vents. Flow velocity near the spacecraft varies from $3 \mathrm{~m} / \mathrm{s}$ at the top deck of the top satellite, to $0.2 \mathrm{~m} / \mathrm{s}$ in the lowest position. In the venting case, the flow velocity is less than $0.1 \mathrm{~m} / \mathrm{s}$ around the top satellite and about $0.6 \mathrm{~m} / \mathrm{s}$ on spacecraft surfaces close to the vents. A large recirculation region is visible in the conical section of the fairing for the ground air conditioning case. Although this region is expected to migrate with time, for simplicity, we assumed that the flow is steady. The same assumption was made in the case of the venting flow. Although no data was available to validate the flow results, the results agree qualitatively with fairing analysis of the Delta II ELV performed by Kandula and Walls ${ }^{11}$.
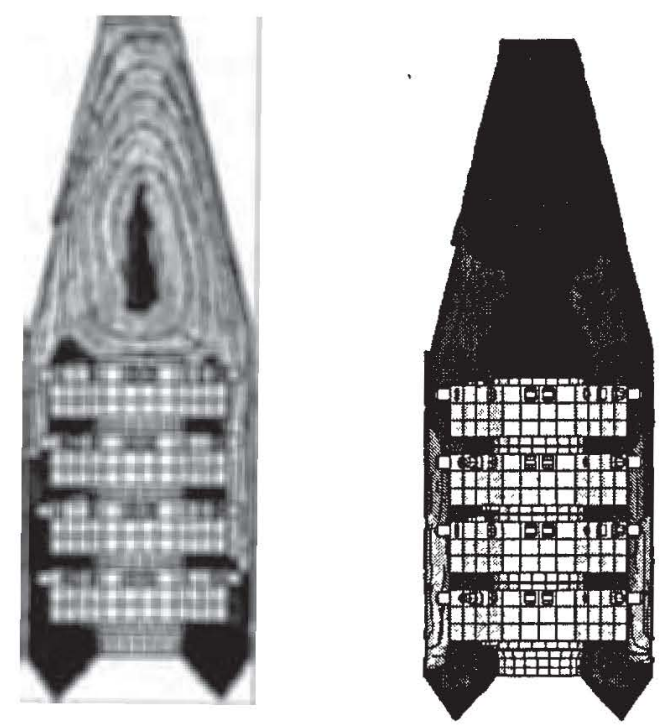

Figure 7. Velocity contours and few selected streamlines for the ground air conditioning and ascent payload venting case

\subsection{Particle Properties}

The view factor analysis was performed for three sizes of particles: $50 \mu \mathrm{m}, 100 \mu \mathrm{m}$ and $200 \mu \mathrm{m}$. The particle density was set to vary from $1550 \mathrm{~kg} / \mathrm{m}^{3}$ to $2700 \mathrm{~kg} / \mathrm{m}^{3}$ (cotton to aluminum), and the particles were injected with initial velocity in the 0.1 to $1.0 \mathrm{~m} / \mathrm{s}$ range. Coefficient of restitution ranged from 0.1 to 0.3 and the sticking velocity range was 0.1 to 0.5 $\mathrm{m} / \mathrm{s}$. Material properties of each launched particle (analysis "ray") were determined by randomly selecting a value from each property range. Ten particles were launched from each source element. In this work we did not directly account for the variation in material interaction between different material pairs, due to lack of available data on this subject.

\section{RESULTS}

Figure 8 shows particle traces for the four considered cases for contaminants originating on the fairing. The three colors correspond to the particle sizes considered in our analysis. As expected, particles are seen to follow highly non-linear and non-uniform paths. In case 1, the on-ground air-conditioning, particle dynamics is dominated by the applied flow field. A large number of particles originating in the upper conical section is trapped in the circulating region. Somewhat surprising is not finding a significant variation in particle trajectories for the three different particle sizes. However, by considering the ratio of the applied forces, we can see that in the conical section, the magnitude of the drag force exceeds the gravitational force by at least an order of magnitude even for the largest particles. Some particle mass effect is however noticeable near the upper deck. The lightest particles continue to follow the streamlines, while the heavier particles settle on the surface. The highest concentration of particles is seen $180^{\circ}$ from the inlet.

The particle concentration is more uniform for the launch and ascent venting cases due to the azimuthal uniformity of the applied forces. The top deck of the top satellite has the highest view factor of the fairing, but even areas with no direct line of sight, such as the spaces between the satellites, are seen to contain particles. The last case considers the effect of vehicle tilt on particulate transport. As expected, particle concentration is governed by the direction of the 
acceleration vector. Figure 9 shows the view factors calculated from the particle traces above. The satellites are rotated about $15^{\circ}$ from the view shown in the previous plot. This plot shows the average viewfactor for the three particle sizes. Results for case 2 and 3 show maximum and uniform deposition on the top deck of the top spacecraft. These results are expected given the azimuthally uniform applied force field in these cases. Results for the burn out case are shown in the last image. Largest viewfactors are seen near the top deck HPCA, which corresponds to the side of the launch vehicle facing towards the ground.
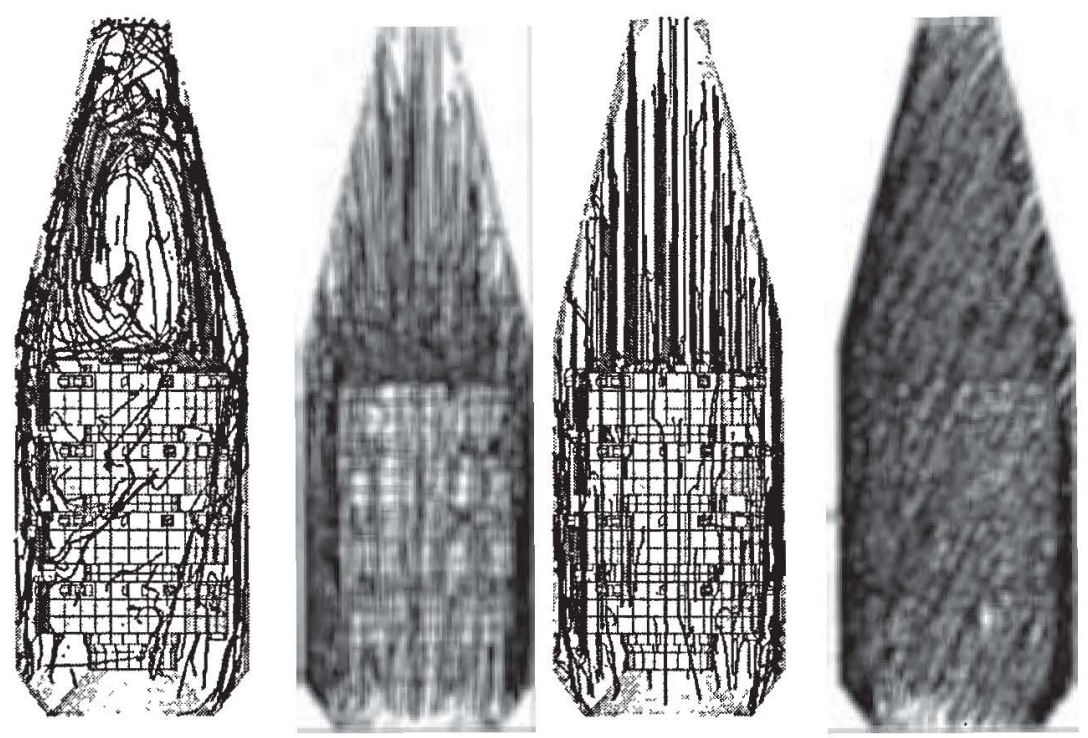

Figure 8. Particle trajectories for ground, launch, venting and burnout, respectively. Colors correspond to the particle bins: red: $50 \mu \mathrm{m}$, blue: $100 \mu \mathrm{m}$, black: $200 \mu \mathrm{m}$
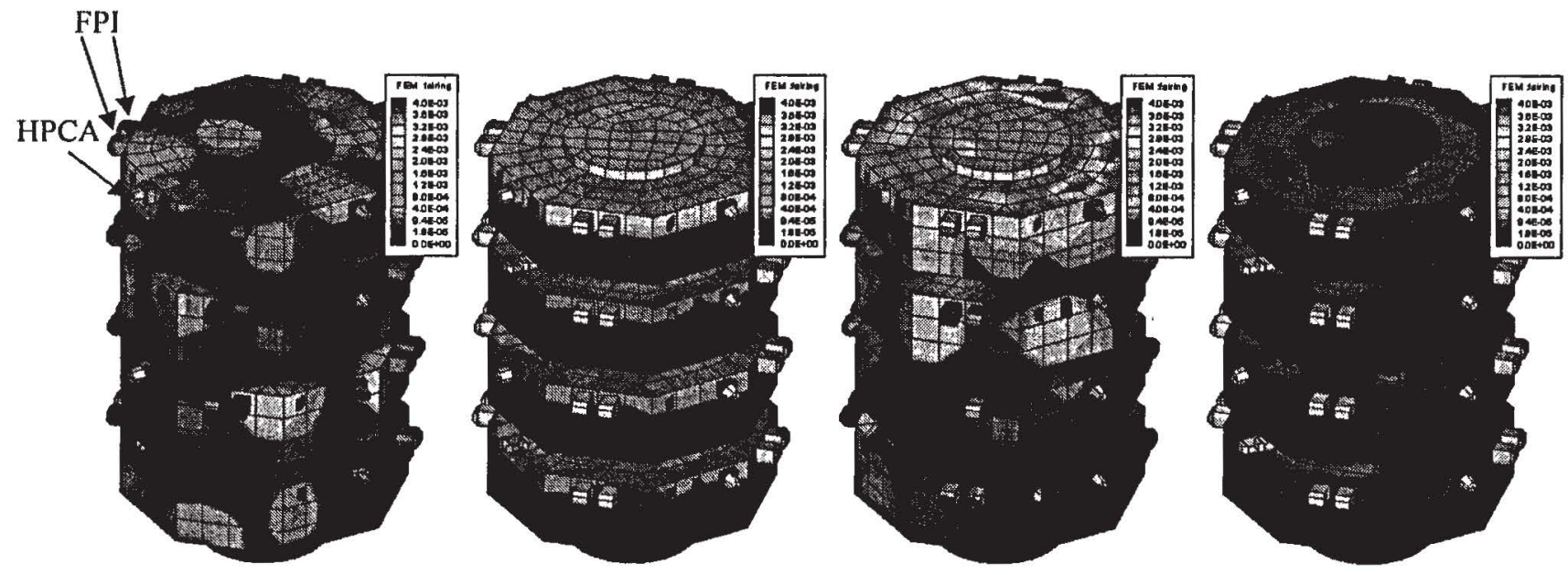

Figure 9.Combined view factors from fairing for the 4 studied cases 


\section{SURFACE CONTAMINATION ANALYSIS}

The current version of Mastram does not include capability to automatically compute contamination levels given initial distribution and detachment model. As such, this calculation must performed manually as part of post-processing for all surfaces of interest. Here we have considered two instruments, the top deck HPCA and the top-deck FPI located clockwise from the HPCA. These instruments correspond to the highest contamination levels and as such can be used to bracket the expected fallout. Number of particles of certain size arriving at target $t$ given $n_{s}$ particles leaving the source can be calculated from

$$
n_{t}=n_{s} f \frac{A_{s}}{A_{t}}
$$

where $A_{s}$ and $A_{t}$ are the areas of the source and target. For these two instruments, fallout is limited to particles originating in the conical section of the fairing, area of which is computed from geometry. The area of the target and the view factor $f$ are obtained from the computational model. To simplify the analysis, we have assumed that there are no significant differences between the behavior of particles of different sizes, and the view factor was calculated by taking a simple average between the three results. This assumption is confirmed by the small differences visible in particle trace traces.

The view factors on the HPCA and the FPI for Case 1 were 0.003 and 0.0005 , respectively. Detachment probability was calculated by including the mean value of $5 \mathrm{~m} / \mathrm{s}$ of near surface air flow in the conical section of the fairing. The size distribution of particles landing on the two instruments is plotted in Figure 10 a). The solid line indicates counts of particles of various sizes existing on the fairing assuming CL 300 and slope 0.926 . The two dashed lines show the accumulation of particulates from the fairing on the apertures of the two instruments.

The slope of the IEST-1246D profile is seen experimentally to decay with time. While the 0.926 slope corresponds to freshly cleaned surfaces, levels approaching 0.3 are common on surfaces that have been left exposed for many days ${ }^{4}$. By matching the obscured areas, an older surface is seen to contain higher concentration of large particles than a corresponding freshly-cleaned surface. This effect could be due to conglomeration of smaller particles into larger globules. To account for this change, we assumed that the slope decreased to 0.5 by the time of launch. The initial distribution of particles on the fairing at launch was obtained directly from the IEST-1246D profile for cleanliness level with total obscuration area corresponding to the particles remaining on the fairing after the $\mathrm{A} / \mathrm{C}$ flow was applied. Figure $10 \mathrm{~b}$ ) shows results for this case. The view factors were 0.0007 and 0.0006 , respectively. This plot shows increased concentration of large particles, which is a direct result of the change in the particle size distribution model on the fairing.

Similar analysis was performed for the two remaining cases. For the case of ascent venting, we retained the 0.5 slope, and the initial distribution was obtained by subtracting particles detached during launch from the at-launch distribution. Result for this case is seen in Figure $10 \mathrm{c}$ ). The detachment probability was calculated by assuming total $3.4 \mathrm{~g}$ applied load, corresponding to the vehicle axial thrust. Air flow in the conical section was negligible in this case and was not included in the detachment calculation. The view factors were 0.00008 and 0.0004 , respectively. The last plot shows results for the max-load case. This case resulted in very large view factors, 0.002 and 0.001 for HPCA and FPI, respectively. These large magnitudes are directly related to the inclination of the launch vehicle, which was selected to generate the largest concentration of particles in the vicinity of these particles. Particles originating on the opposite side of the spacecraft are accelerated towards the fairing below the instruments, and bounce off towards the instruments. The actual behavior in a real system will be altered by the presence of acoustic blankets, which can be assumed to absorb the impacting particles. In addition, the transport mechanics will be altered by the actual azimuthal orientation of the spacecraft relative to the ground direction.

Despite the large view factors computed in this case, the actual contamination risk is very minimal due to depletion of particles in the previous cases. From the results, we can see that all particles larger than $100 \mu \mathrm{m}$ have detached by the time the fairing experiences venting. The dashed lines, showing the accumulated concentration of particles, show only minimal change in Case 3 and 4, with the variation limited to sub $50 \mu \mathrm{m}$ particles. Prediction of the final contamination levels on the instruments requires taking into account the transport of contaminants on the satellites. This analysis was not performed, instead we assumed that the contaminants on the spacecraft remain in their prelaunched configuration. The final contamination can then be calculated by combining the accumulated fallout from the fairing with the initial cleanliness level on the spacecraft. By matching the areas obscured by the particles, the final cleanliness levels can be computed as 385 for the HPCA and 380 for the FPI. The initial 0.926 slope was used in this final calculation. 


\section{- REVIEW COPY - JULY 082010}
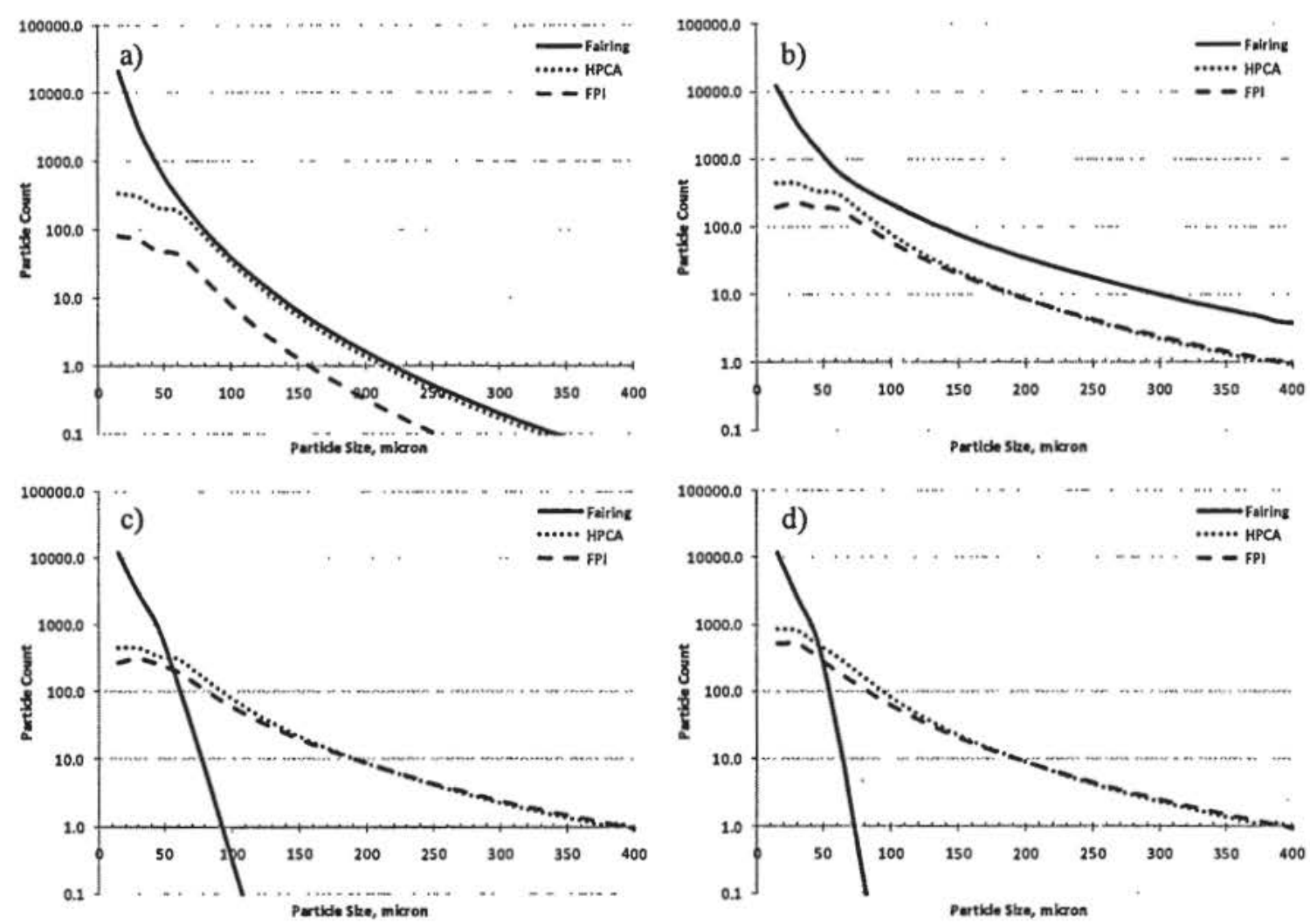

Figure 10. Particle counts existing on fairing and particle accumulation on the two studied instruments for ground $\mathrm{A} / \mathrm{C}$, launch, venting and max load, respectively.

\section{ACKNOWLEDGMENTS}

This work was sponsored by NASA Project NNG07CA21C. The first author would also like to acknowledge helpful discussions with Tim Gordon and Tony Dazso.

\section{REFERENCES}

1. Solving Magnetospheric Acceleration, Reconnection and Turbulence (SMART), http://mms.space.swri.edu

2. J. Gimhold and S. Barabash, "Contamination and cleanliness control of the Mars Express ASPERA-3 experiment during AIV and pre-launch activities", Technical Report MEX-ASP-TN-990716-1, 1999

3. C.W. Carlson, et.al., "An instrument for rapidly measuring plasma distribution functions with high resolution', Adv. Space Res., 2, 7, 1982

4. R. A. Crook, "Estimating particle redistribution from launch loads", J. Optical Engineering, 45, 059001, 2006

5. A. Klavins and A. L. Lee, "Spacecraft particulate contaminant redistribution", Optical system contamination: Effects, measurement, control, 777, pp. 236-244, 1987

6. A.C. Hindmarsch, "ODEPACK, a systematized collection of ODE solvers", Scientific computing: applications of mathematics and computing to the physical sciences, 1, pp 55-64, 1983

7. F.M. White, Viscous Fluid Flow, McGraw-Hill, New York, 1991

8. D. Hughes, US Patent Application 2009/0312994-A1, 2009

9. R. J. Renka, "Multivariate interpolation of large sets of scattered data," ACM Transactions on Mathematical Software (TOMS), 14, No. 2, pp 139-148

10. Atlas Launch System Mission Planner's Guide, Rev 10a, January 2007, http://www.lockheedmartin.com/ssc/commercial_launch_services/MissionPlannersGuide.html

11. M. Kandula and L. Walls, "An Application of Overset grids to Payload/Fairing Internal Flow CFD Analysis", $20^{\text {th }}$ AIAA Applied Aerodynamics Conference, St. Louis, MS, 24-26 June, 2002, AIAA-2002-3253 